\title{
Diseño de un sistema de control avanzado para regular la velocidad de una turbina de vapor acoplada a un generador DC
}

\section{Advanced controller design for speed control in a steam turbine coupled to DC generator}

\author{
Hernando González-Acevedo \\ M.Sc. en Ingeniería \\ Universidad Autónoma de Bucaramanga \\ Bucaramanga, Colombia \\ hgonzalez7@unab.edu.co
}

\author{
Hernan González-Acuña \\ M.Sc. en Ingeniería \\ Universidad Autónoma de Bucaramanga \\ Bucaramanga, Colombia \\ hgonzalez3@unab.edu
}

\begin{abstract}
Resumen- El artículo presenta la metodología para el diseño de dos estrategias de control, LQG (Linear Quadratic Gaussian Control) y DMPC (Discrete Time Model Predictive Control), para regular la velocidad de una turbina de vapor acoplada a un generador DC de excitación independiente. La dinámica del sistema es representada por un modelo lineal en el cual los parámetros del modelo se calculan a partir de un algoritmo de optimización. Las estrategias de control se implementan en el sistema de control distribuido (DCS), Delta V. El objetivo es mantener constante la velocidad ante variaciones de la presión en la tubería de vapor y modificación de la resistencia del bobinado de campo del generador DC.
\end{abstract}

Palabras clave- Turbina de vapor, generador DC, control LQG, control DMPC.

Abstract- The paper presents the methodology for the design of two control strategies, LQG (Linear-Quadratic-Gaussian Control) and DMPC (Discrete Time Model Predictive Control), for speed control in a steam turbine coupled to separately excited DC generator. The dynamics of the system is represented by a linear model in which the model parameters are calculated using an optimization algorithm. The control strategies were implemented on a distributed control system (DCS), Delta V. The goal is to maintain the speed constant despite the variation of pressure in the steam pipeline and changes in the field resistance of the DC generator.

Keywords- Steam turbine, DC generator, LQG control, DMPC control.

\section{INTRODUCCIÓN}

Una turbina de vapor es una turbomáquina que transforma la energía de un flujo de vapor en energía mecánica, a través de un intercambio de cantidad de movimiento entre el fluido de trabajo (vapor) y el rodete, que cuenta con palas o álabes para poder realizar el intercambio energético. Las turbinas de vapor están presentes en diversos ciclos de potencia que utilizan un fluido que pueda cambiar de fase, entre estos el más importante es el ciclo de rankine, el cual genera el vapor en una caldera, de la que sale en unas condiciones de elevada temperatura y presión. En la turbina se transforma la energía interna del vapor en energía mecánica, que, típicamente, es aprovechada por un generador para producir electricidad. Los modelos dinámicos y estáticos de la turbina de vapor se han venido desarrollando de diferentes formas, a través de procesos similares. Los modelos incluyen la unidad de recalentamiento, la cual es representada como una función de transferencia de primer orden y los parámetros del modelo se ajustan a partir de datos experimentales [1]. Otros modelos trabajan funciones lineales de primer orden y funciones no lineales [2], que relacionan el caudal con las diferencias de presión, los parámetros del modelo son determinados a partir de la respuesta dinámica de una planta de 600 
MW ante perturbaciones aplicadas, utilizando un algoritmo genético.

La variable por regular, comúnmente en estos procesos, es la velocidad del generador acoplado a la turbina. Dado que las funciones lineales y no lineales que presentan los modelos clásicos no describen completamente la dinámica del sistema, algunas estrategias se centran en modificar en tiempo real las constantes de un control PID. Entre esas estrategias está el regulador PID adaptativo [3], es decir, un sistema difuso modifica las constantes proporcional, integral y derivativa de acuerdo con la señal de error y razón de cambio del error. Esta metodología ha sido optimizada utilizando cuatro métodos diferentes: algoritmos genéticos, optimización por enjambre de partículas (PSO), optimización por enjambre de bacterias (BPSO) y controladores neurodifusos (ANFIS) [4]. En [5] se compara la robustez de un control predictivo GPC (Generalized Model Predictive Control) con un PID, para regular la potencia de una unidad turbina-caldera y se demuestra que el control predictivo presenta buen seguimiento a la señal de referencia y rechazo a perturbaciones comparado con el PID. La referencia [6] compara la respuesta dinámica de un control predictivo GPC y CRHPC (Constrained Receding-Horizon Predictive Control), presentando esta última mejor exactitud y menores fluctuaciones ante perturbaciones aplicadas a la turbina. Otra técnica empleada ha sido el control LQG (Linear-quadratic-Gaussian) para regular la temperatura de vapor de una planta de $300 \mathrm{Mw}$ [7]. Los resultados se comparan con los obtenidos con un controlador PID, y se demuestra que el rendimiento del LQG es mejor, especialmente, durante los transitorios.

\section{MODELO DINÁMICO}

La Universidad Autónoma de Bucaramanga cuenta en su laboratorio de planta piloto con una turbina de vapor, tipo Terry, acoplada a un generador de corriente continua de excitación independiente de $1 \mathrm{Kw}$. El vapor es suministrado por una caldera pirotubular de $10 \mathrm{BHP}$, a una temperatura de 170 Celsius, con una capacidad máxima de $345 \mathrm{Lb} /$ hora. La caldera tiene un controlador onoff que regula la presión de vapor, la cual oscila en un rango entre 115 a 125 psi. El flujo másico es regulado a través de una válvula proporcional, marca Spirax Sarco.

\subsection{Modelo dinámico de la turbina de vapor}

En muchos casos, el modelo dinámico de una turbina de vapor se simplifica; las variables intermedias se omiten y todo queda con base en las variables de entrada y salida [8]. La potencia mecánica desarrollada por una turbina sin secciones de recalentamiento se basa en una ecuación de continuidad (1)

$$
\frac{\partial W}{\partial t}=V \frac{\partial \rho}{\partial t}=F_{\text {in }}(t)-F_{\text {out }}(t)
$$

donde $W$ es la masa del vapor en la turbina $[K g], V$ el volumen de la turbina $\left[\mathrm{m}^{3}\right], \rho$ la densidad del vapor $\left[K_{g} / \mathrm{m}^{3}\right]$ y $F$ el flujo másico de vapor $\left[K_{g} / S\right]$. Suponiendo que el flujo de salida de la turbina es proporcional a la presión en la turbina, de acuerdo con (2)

$$
F_{\text {out }}=P \frac{F_{O}}{P_{O}}
$$

donde $P$ es la presión de vapor en la turbina $\left[K P_{\alpha}\right], P_{O}$ la presión nominal y $F_{O}$ el flujo másico nominal de la turbina. Con temperatura constante en la turbina se tiene (3)

$$
\frac{\partial \rho}{\partial t}=\frac{\partial P}{\partial t} \cdot \frac{\partial \rho}{\partial P}
$$

Unificando las ecuaciones (1), (2), (3) resulta el modelo matemático mostrado en (4) y (5)

$$
\begin{gathered}
F_{\text {in }}(t)-F_{\text {out }}(t)=V \frac{\partial P}{\partial t} \cdot \frac{\partial \rho}{\partial P} \\
=V \frac{\partial \rho}{\partial P} \cdot \frac{P O}{F_{O}} \cdot \frac{\partial F_{\text {out }}}{d t}=T_{T} \frac{\partial F_{\text {out }}}{d t} \\
T_{T} \frac{\partial F_{\text {out }}}{d t}+F_{\text {out }}(t)=F_{\text {in }}(t)
\end{gathered}
$$

Al aplicar la transformada de Laplace, la función de transferencia de una turbina de vapor se expresa como (6)

$$
H(S)=\frac{F_{\text {out }}(S)}{F_{\text {in }}(S)}=\frac{1}{T_{T^{S+1}}}
$$

donde $T_{T}=V \frac{\partial \rho}{\partial P} \cdot \frac{P_{O}}{F_{O}}$ es una constante de tiempo [sg]. El torque de la turbina es proporcional al flujo másico de vapor y se muestra en (7)

$$
T_{m}(t)=k_{1} \cdot F_{\text {out }}(t)
$$

donde $k_{1}$ es una constante proporcional. El flujo másico de vapor se controla por medio de una válvula proporcional, cuya dinámica se define en (8), donde $k_{2}$ es un constante de proporcionalidad, el retardo de la válvula y $T_{d}$ el porcentaje de apertura de la válvula. 


$$
F_{\text {in }}(S)=k_{2} e^{-T_{d}^{s}} u(S)
$$

A partir de las ecuaciones (6), (7) y (8) la función de transferencia que caracteriza la dinámica de la turbina se define como (9)

$$
G T(S)=\frac{T_{m}(S)}{u(S)}=\frac{k_{1} k_{2} e^{-T_{d^{s}}}}{T_{T^{S+1}}}
$$

\section{2 Modelo dinámico del generador DC de excitación independiente}

El generador DC de excitación independiente presenta la ventaja que el campo del estator es constante al no depender de la carga del motor, y el par de fuerza es prácticamente constante [9]. Las variaciones de velocidad al aumentar la carga se deberán solo a la disminución de la fuerza electromotriz por aumentar la caída de tensión en el rotor. La dinámica de esta clase de máquinas está representada por (10), (11) y (12)

$$
\begin{gathered}
V_{f}(t)=R_{f} I_{f}(t)+L_{f} \frac{d I_{f}(t)}{d t} \\
K_{v} \omega(t)=\left(R_{\alpha}+R_{L}\right) I_{\alpha}(t)+L_{\alpha} \frac{d I_{\alpha}(t)}{d t} \\
T_{m}(t)=J \frac{d \omega(t)}{d t}+B_{m} \omega(t) \\
+K_{I} I_{f}(t) I_{\alpha}(t)
\end{gathered}
$$

donde $V_{f}$ es la tensión aplicada a la bobina de campo, $I_{f}$ es la corriente de la bobina de campo, $R_{f}$ la resistencia del bobinado de campo, $L_{f}$ la inductancia del bobinado de campo, $K_{v}$ la constante de fuerza contraelectromotriz, $\omega$ la velocidad del eje del generador DC, $R_{\alpha}$ la resistencia del bobinado de armadura, $L_{\alpha}$ la inductancia del bobinado de armadura, $I_{\alpha}$ la corriente de armadura, $R_{L}$ la resistencia de carga conectada al generador DC, $T_{m}$ el torque que aplica la turbina de vapor al eje del generador, $J$ es la suma de la inercia del generador y el eje de la turbina, $B_{m}$ la constante de fricción viscosa y $K_{I}$ la constante de par. Al despreciar el transitorio en el bobinado de campo debido a que la tensión $V_{f}$ es fija, la función de transferencia que representa el comportamiento dinámico del generador DC se expresa en (13).

$$
\begin{aligned}
& G G(S)=\frac{\omega(S)}{T_{m}(S)}= \\
& \frac{R_{\alpha}+R_{L}+L_{\alpha^{s}}}{\left(J_{S}+B_{m}\right)\left(R_{\alpha}+R_{L}+L_{\alpha^{s}}\right)+K_{i} K_{v} \cdot V_{f} / R_{f}}(13)
\end{aligned}
$$

\subsection{Programación cuadrática secuencial}

El diseño por optimización consiste en la obtención de la solución de un problema, a través de la aplicación de una formulación matemática que permite evaluar y comparar diversas soluciones con el fin de hallar una respuesta óptima. La programación cuadrática secuencial (SQP) es una técnica de optimización clásica basada en el cálculo del gradiente de la función objetivo y de las restricciones. La técnica se divide en dos subproblemas de optimización, uno para determinar la dirección de búsqueda, y otro para calcular el paso de una iteración a otra. En cada iteración se resuelve un problema cuadrático, el cual se establece en función del gradiente de la función objetivo y de las restricciones.

Los siguientes parámetros se conocen a partir de la hoja técnica del generador DC: $R_{f}=367 \Omega$ $L_{f}=20.6 \mathrm{H}, \quad R_{\alpha}=7.4 \Omega, \quad L_{\alpha}=11.38 \mathrm{mH}$, $R_{L}=20.8 \Omega$; el bobinado de campo está excitado a una tensión de 213 voltios DC. Para determinar los parámetros desconocidos del modelo dinámico de la turbina y del generador DC se programa un algoritmo de optimización, fundamentado en la teoría de SQP, aplicando la siguiente metodología:

- Se sintoniza un control proporcional y se registran los datos de la señal de referencia, porcentaje de apertura de la válvula y velocidad del generador, a una tasa de muestreo de un segundo, Fig. 1. La referencia se modifica en un rango de 0 - 1500 rpm, garantizando de esta forma un comportamiento lineal del sistema.

- El modelo dinámico del sistema se implementa en la herramienta de Simulink de Matlab; la señal de entrada al modelo es el porcentaje de apertura de la válvula y la salida de la velocidad de la turbina. En la Fig. 2, se observa el diagrama de bloques de la planta, lo conforma la función de transferencia de la turbina GT y la función de transferencia del generador GG, ecuación (9) y (13), respecti- 
vamente. La constante $\mathrm{K}$ representa el cambio de unidades de la velocidad del generador: de radianes por segundo $(\mathrm{rad} / \mathrm{s})$ a revoluciones por minuto (RPM).

Se programa un algoritmo de optimización en el cual el objetivo es disminuir el error medio cuadrático (14), entre los datos simulados $\left(x_{\text {sim }}\right)$ y los datos experimentales $\left(x_{E x p}\right)$, dado un vector $x$ con los valores de los parámetros del modelo matemático.

$$
f=\sum_{k=0}^{N}\left(x_{\text {sim }}(k)-x_{E x p}(k)\right)^{2}
$$

En la Tabla I se observa los valores de los parámetros, una vez se ejecuta el algoritmo de optimización. En (15) se presenta el modelo dinámico de la turbina de vapor acoplada con el generador DC y en la Fig. 3 se compara la respuesta transitoria del modelo con los datos experimentales.

\section{Fig. 1. DATOS EXPERIMENTALES. A) PORCENTAJE DE APERTURA DE LA} VÁLVULA, B) VELOCIDAD MECÁNICA DE LA TURBINA
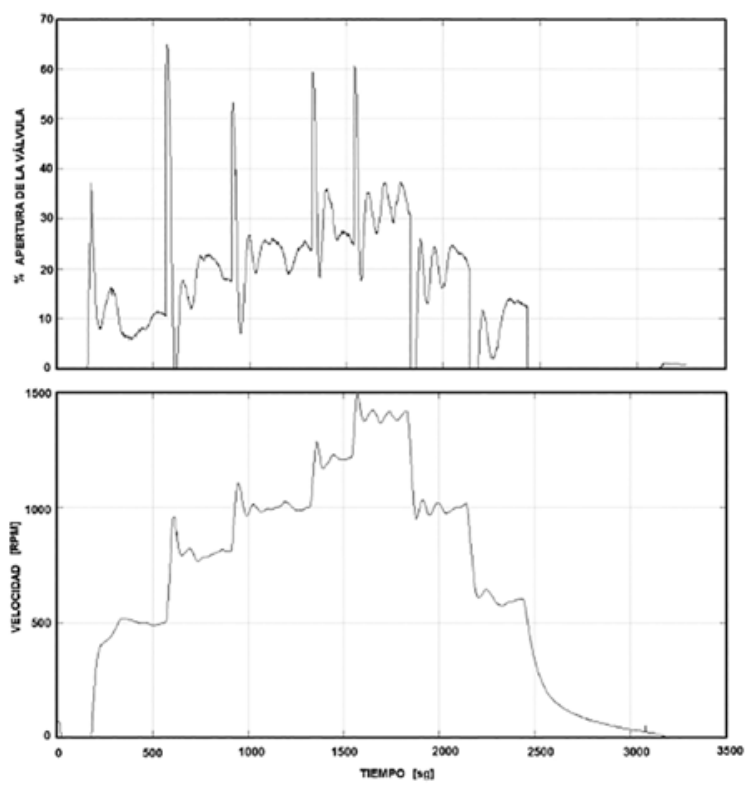

Fuente: Los autores.
TABLA I

PARÁMETROS DEL MODELO MATEMÁTICO

\begin{tabular}{|c|c|}
\hline Parámetro & Valor \\
\hline \multicolumn{2}{|r|}{ Turbina de vapor } \\
\hline$T_{T}$ & $119.7[s g]$ \\
\hline$K_{1} * K_{2}$ & $0.055031[\mathrm{Nm} / \%]$ \\
\hline$T_{d}$ & $9[s g]$ \\
\hline \multicolumn{2}{|r|}{ Generador DC } \\
\hline$J$ & $0.006015527721586\left[\mathrm{Kg} \cdot \mathrm{m}^{2}\right]$ \\
\hline$K_{i}$ & $0.843524807917543\left[N \cdot m / A^{2}\right]$ \\
\hline$K_{v}$ & $0.620330916257256\left[N \cdot m / A^{2}\right]$ \\
\hline$B_{m}$ & $0.000922377688340\left[\frac{\mathrm{N} \cdot \mathrm{m}}{\mathrm{rad} / \mathrm{sg}}\right]$ \\
\hline
\end{tabular}

Fuente: Los autores.

$G(S)=\frac{(0.01879 S+46.56) e^{-9 s}}{0.02574 S^{3}+63.8 S^{2}+124.5 S+1.036}($

Para simplificar (15) se aproxima el retardo que presenta la función de transferencia de la planta por una función de transferencia de primer orden (16), obteniendo una nueva función de transferencia (17).

$$
e^{-9 s} \approx \frac{-S+1 / 9}{S+1 / 9}
$$

$G(S)=\frac{-0.01879 S^{2}-46.56 S+10.35}{0.02574 S^{4}+63.81 S^{3}+138.7 S^{2}+28.7 S+0.2302}(17)$

Fig. 2. DIAGRAMA DE BLOQUES DEL MODELO DINÁMICO DE LA PLANTA

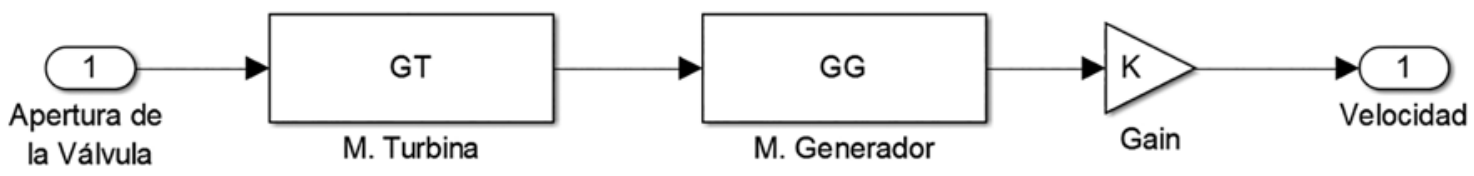

Fuente: Los autores. 
Fig. 3. COMPARACIÓN DE LOS DATOS EXPERIMENTALES CON LOS DATOS DE SIMULACIÓN PARA LA SEÑAL DE VELOCIDAD

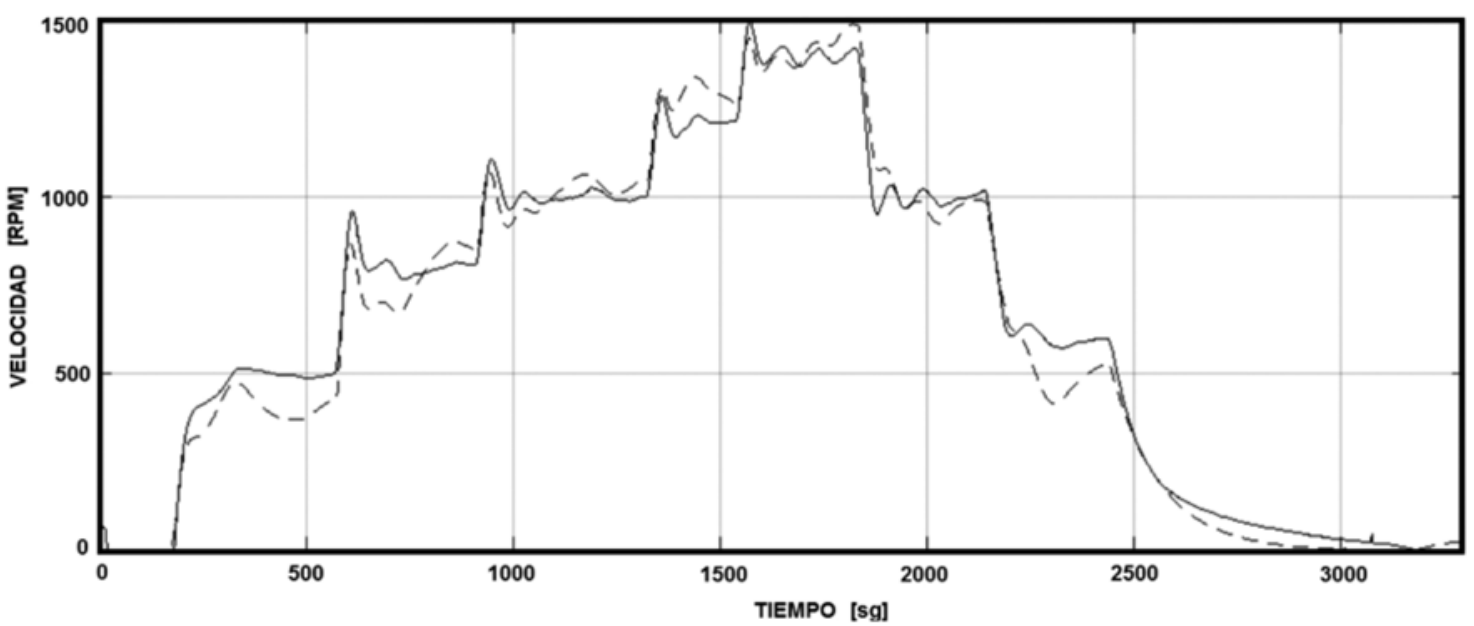

Fuente: Los autores.

La dinámica del sistema se representa en un modelo en espacio de estados discretos, asumiendo un período de muestreo de un segundo (18).

$$
x(k+1)=G_{L} x(k)+H_{L} u(k)
$$

$$
y(k)=C_{L} x(k)
$$

Donde,

$$
G_{L}=\left[\begin{array}{cccc}
-0.000117 & -0.004559 & -0.000102 & -0.000003 \\
0.001424 & 0.055065 & -0.042968 & -0.001370 \\
0.039239 & 1.519927 & 0.880884 & -0.003809 \\
0.006815 & 0.264122 & 0.238425 & 0.999629
\end{array}\right]
$$

$$
\begin{aligned}
& H_{L}=\left[\begin{array}{l}
0.000089 \\
0.039239 \\
0.109044 \\
0.010605
\end{array}\right] \\
& C=\left[\begin{array}{llll}
0 & -0.002851 & -1.766304 & 1.570189
\end{array}\right]
\end{aligned}
$$

\section{DISEÑO DEL SISTEMA DE CONTROL}

La turbina de vapor es el elemento con mayor importancia para la generación de la energía eléctrica en centrales térmicas. Para que la energía eléctrica generada en dichas centrales sea prácticamente constante, se hace necesario tener un sistema de regulación de la velocidad de la turbina de vapor con el fin de mantener la velocidad de giro de los rotores del conjunto turbina-generador lo más cercano posible al valor de referencia.

\subsection{Sistema de seguimiento}

Para funciones de transferencia que no presentan integrador, el principio básico del diseño de un sistema de seguimiento es insertar un integrador en el camino directo entre el comparador de error y la planta [10], tal como se observa en la Fig. 4.

Fig. 4. DIAGRAMA DE BLOQUES DEL SISTEMA DE SEGUIMIENTO

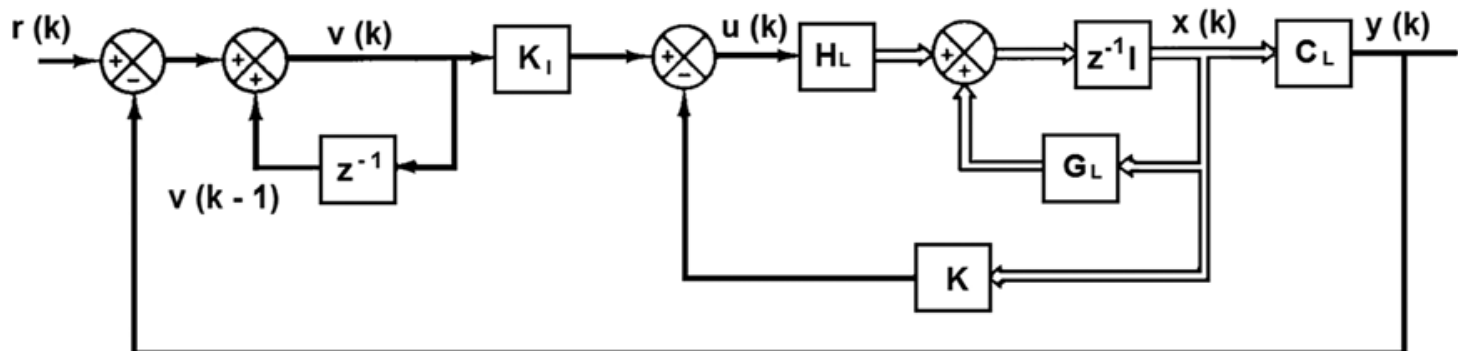


Asumiendo que la entrada de referencia (función escalón) se aplica en $t=0$, la dinámica del sistema para $t>0$ está descrita por (19)

$$
\begin{aligned}
& {\left[\begin{array}{l}
x(k+1) \\
v(k+1)
\end{array}\right]=\left[\begin{array}{cc}
G_{L} & 0 \\
-C_{L} G_{L} & 1
\end{array}\right]} \\
& {\left[\begin{array}{l}
x(k) \\
v(k)
\end{array}\right]+\left[\begin{array}{c}
H_{L} \\
-C_{L} H_{L}
\end{array}\right] u(k)+} \\
& {\left[\begin{array}{l}
0 \\
1
\end{array}\right] r(k+1)}
\end{aligned}
$$

Considerando que $r(k)$ es una entrada escalón, se tiene que $r(k)=r(k+1)=r$ (constante). Cuando $k$ tiende a infinito, la ecuación de estados se define como (20)

$$
\begin{aligned}
& {\left[\begin{array}{c}
x(\infty) \\
v(\infty)
\end{array}\right]=\left[\begin{array}{cc}
G_{L} & 0 \\
-C_{L} G_{L} & 1
\end{array}\right]\left[\begin{array}{c}
x(\infty) \\
v(\infty)
\end{array}\right]+} \\
& {\left[\begin{array}{c}
H_{L} \\
-C_{L} H_{L}
\end{array}\right] u(\infty)+\left[\begin{array}{l}
0 \\
1
\end{array}\right] r(\infty)}
\end{aligned}
$$

Restando (19) de (20), se obtiene (21) y (24) se transforman en (26) y (27)

$$
\begin{aligned}
& {\left[\begin{array}{c}
x_{e}(k+1) \\
v_{e}(k+1)
\end{array}\right]=\left[\begin{array}{cc}
G_{L} & 0 \\
-C_{L} G_{L} & 1
\end{array}\right]\left[\begin{array}{c}
x_{e}(k) \\
v_{e}(k)
\end{array}\right]} \\
& +\left[\begin{array}{c}
H_{L} \\
-C_{L} H_{L}
\end{array}\right] u_{e}(k)
\end{aligned}
$$

Donde,

$$
\begin{gathered}
x_{e}(k)=x(k)-x(\infty) \\
v_{e}(k)=v(k)-v(\infty) \\
u_{e}(k)=-K x_{e}(k)+K_{I} v_{e}(k)
\end{gathered}
$$

Se define un nuevo vector de estados, $\xi(k)$, de dimensión $(n+1)$, mediante (25)

$$
\xi(k)=\left[\begin{array}{l}
x_{e}(k) \\
v_{e}(k)
\end{array}\right]=(n+1)-v e c t o r
$$

Así, (21) y (24) se transforman en (26)

$$
\begin{gathered}
\xi(k+1)=\hat{G} \xi(k)+\hat{H} u_{e}(k) \\
u_{e}(k)=\hat{K} \xi(k)
\end{gathered}
$$

Donde,

$$
\hat{G}=\left[\begin{array}{cc}
G_{L} & 0 \\
-C_{L} G_{L} & 1
\end{array}\right] \quad \hat{H}=\left[\begin{array}{c}
H_{L} \\
-C_{L} H_{L}
\end{array}\right] \quad \hat{K}\left[\begin{array}{ll}
K & -K_{I}
\end{array}\right]
$$

\subsection{Control LQG (Linear Quadratic Gaussian Control)}

El controlador LQG es la combinación de una ganancia de realimentación de estados y un estimador de estados, tipo Kalman [11]. El primer paso para el diseño del controlador LQG es determinar una matriz de ganancias $\hat{K}$ que minimice la función de costo $J$, (28). Las matrices $Q$ y $R$ son matrices definidas positivas, generalmente diagonales, que determinan el equilibrio entre el seguimiento de una trayectoria por parte de los estados y la energía de la señal control requerida por el sistema para alcanzar los objetivos de control deseados. La matriz $\hat{K}$ se determina resolviendo la ecuación matricial de Riccatti, dadas las matrices $\hat{G}, \hat{H}, Q$ y $R$.

$$
J=\frac{1}{2} \sum_{k=0}^{\infty}\left(\xi^{T} Q \xi+u_{e}^{T} R u_{e}\right)
$$

Para estimar los estados se utiliza un filtro Kalman, el cual asume la presencia de ruido gaussiano en la señal de control y la variable a medir en el proceso. Conocidas las varianzas del ruido de estas dos señales, $Q_{n}$ y $R_{n}$, se determina un vector de ganancias $L$ que minimiza el error en la estimación, $x-\hat{x}$, utilizando la ecuación de Ricatti. Matemáticamente, los estados se calculan a partir de la siguiente expresión (29)

$$
\begin{aligned}
& \hat{x}(k+1)=G_{L} \hat{x}(k)+H_{L} u(k) \\
& +L\left(y(k)-C_{L} \hat{x}(k)\right)
\end{aligned}
$$

donde $u(k)$ es la señal de control y $y(k)$ la variable a controlar del proceso. Los siguientes valores se asumieron para el diseño del controlador: $Q=\operatorname{diag}(1,1,1,8.5,0.009)$ y $R=5$. Al solucionar la ecuación de Ricatti se obtiene $\hat{K}=[0.061111$, $, 2.367096,1.369298,1.786684,-0.037269]$. Para el diseño del observador se asumen las siguientes varianzas: $Q_{n}=176.9876$ y $R_{n}=21225$, a partir de estos valores se obtiene la matriz $L=10^{-8}$ [37.59397, $\left.-1129.21097,-51464.655,1617969.98\right]$

La Fig. 5 muestra el diagrama de bloques de la estrategia de control LQG. Si la señal de control alcanza los límites prefijados del actuador, al utilizar un controlador con acción integral, el error continuará incrementándose, esto significa que el término integral puede volverse muy grande y producirse el efecto llamado windup, esto genera un 
Fig. 5. DIAGRAMA DE BLOQUES DEL CONTROLADOR LGQ CON GANANCIA ANTIWINDUP

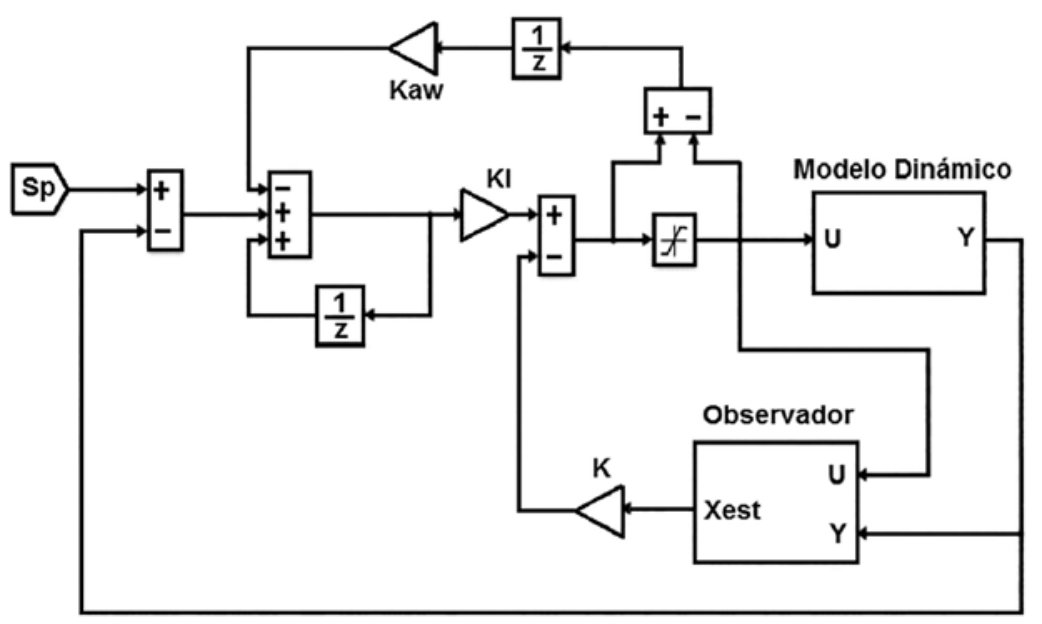

Fuente: Los autores.

gran sobrepaso, mayor tiempo de establecimiento y algunas veces inestabilidad en la respuesta transitoria. Back calculation es un método que permite compensar este fenómeno [12], el cual funciona de la siguiente forma: cuando la salida del actuador se satura, la acción integral es recalculada a través de una ganancia antiwindup $K_{\alpha}$, de forma que el nuevo valor aporte una salida en el límite de saturación. Para el controlador LQG se ajusta una ganancia $K_{\alpha}=10$. Al evaluar la respuesta transitoria del sistema en lazo cerrado a una entrada escalón unitario se obtiene un tiempo de establecimiento de 130 segundos con un sobrepaso del cero por ciento.

\subsection{Control DMPC (Discrete Time Model Predictive Control)}

Una de las propiedades del controlador DMPC es su formulación abierta, que permite la incorporación de distintos tipos de modelos de predicción, sean lineales o no lineales, monovariables o multivariables, y la consideración de restricciones sobre las señales del sistema. Entre las ventajas se puede destacar:

- Formulación en el domino del tiempo, flexible, abierta e intuitiva.

- La ley de control responde a criterios óptimos.

- Permite la incorporación de restricciones en la síntesis del controlador.

Partiendo de un modelo de una planta en espacio de estados en tiempo discreto, dado por(18), se pue- de establecer un nuevo modelo en espacio de estados, definiendo $\Delta x_{m}(K)=x_{m}(k)-x_{m}(k-1)$ y $\Delta u(k)=u(k)-u(k-1)$ dado por (30).

$$
\begin{aligned}
& {\left[\begin{array}{c}
\Delta x_{m}(k+1) \\
y(k+1)
\end{array}\right]=\left[\begin{array}{cc}
G_{L} & 0 \\
C_{L} G_{L} & 1
\end{array}\right]\left[\begin{array}{c}
\Delta x_{m}(k) \\
y(k)
\end{array}\right]} \\
& +\left[\begin{array}{c}
H_{L} \\
C_{L} H_{L}
\end{array}\right] \Delta u(k) \\
& y(k)=\left[\begin{array}{ll}
0 & 1
\end{array}\right]\left[\begin{array}{c}
\Delta x_{m}(k) \\
y(k)
\end{array}\right]
\end{aligned}
$$

Basado en el modelo de espacio de estados descrito en (30), las variables de estado futuras son calculadas secuencialmente a través de (31)

$$
\begin{aligned}
& x\left(k_{i}+N_{p} \mid k_{i}\right)=A^{N_{p}} x\left(k_{i}\right)+A^{N_{p-1}} B \Delta u\left(k_{i}\right) \\
& +A^{N_{p-2}} B \Delta u\left(k_{i}+1\right)+\cdots \\
& +A^{N_{p-N_{c}}} B \Delta u\left(k_{i}+N_{c}-1\right)
\end{aligned}
$$

Donde,(32) se define como

$A=\left[\begin{array}{cc}G_{L} & 0 \\ C_{L} G_{L} & 1\end{array}\right] \quad B=\left[\begin{array}{c}H_{L} \\ C_{L} H_{L}\end{array}\right] \quad C=\left[\begin{array}{ll}0 & 1\end{array}\right]$

$N_{c}$ y $N_{p}$ son los horizontes de control y predicción, respectivamente. A partir de las variables de estado se calcula las variables de salida, como (33)

$$
\begin{aligned}
& y\left(k_{i}+N_{p} \mid k_{i}\right)=C A^{N_{p}} x\left(k_{i}\right)+C A^{N_{p-1}} B \Delta u\left(k_{i}\right) \\
& +C A^{N_{p-2}} B \Delta u\left(k_{i}+1\right) \\
& +\cdots+C A^{N_{p-N_{c}}} B \Delta u\left(k_{i}+N_{c}-1\right)
\end{aligned}
$$


La trayectoria de control $\Delta u$ se representa como la respuesta al impulso de un sistema estable dado por (34)

$$
\Delta u(k)=L(k) \eta
$$

donde $L(k)$ es una matriz constante que representa las funciones de Laguerre y $\eta$ es un vector con los coeficientes de Laguerre [13]. La matriz $L(k)$ contiene $N$ funciones de Laguerre expresados en forma de vectores, como (35)

$$
L(k)=\left[l_{1}(k) l_{2}(k) \cdots l_{N}(k)\right]
$$

La construcción de la matriz $L(k)$ se basa en la siguiente ecuación de diferencias (36)

$$
L(k+1)=A_{l} L(k)
$$

donde la matriz $A_{l}(N x N)$ es función de los parámetros $\alpha$ y $\beta=\left(1-\alpha^{2}\right)$. La condición inicial para la construcción de la matriz $L(k)$ es dada por (37)

$$
L(0)^{T}=\sqrt{\beta}\left[1-\alpha \alpha^{2}-\alpha^{3} \cdots(-\alpha)^{N-1}\right]
$$

En (38) se observa un ejemplo de las matrices $A_{l}$ y $L(0)^{T}$ para $N=5$. Para la construcción de la matriz $L(k)$ se debe definir el número $N$ de funciones de Laguerre por utilizar y el parámetro $\alpha$ (también llamado factor de escalamiento), el cual su valor debe estar entre $0 \leq \alpha<1$. Una vez construida la matriz $L(k)$, esta será constante para el resto del programa.

$$
A_{l}=\left[\begin{array}{ccccc}
\alpha & 0 & 0 & 0 & 0 \\
\beta & \alpha & 0 & 0 & 0 \\
-\alpha \beta & \beta & \alpha & 0 & 0 \\
\alpha^{2} \beta & -\alpha \beta & \beta & \alpha & 0 \\
-\alpha^{3} \beta & \alpha^{2} \beta & -\alpha \beta & \beta & \alpha
\end{array}\right] L(0)=\sqrt{\beta}\left[\begin{array}{c}
1 \\
-\alpha \\
\alpha^{2} \\
-\alpha^{3} \\
\alpha^{4}
\end{array}\right]
$$

Para un sistema SISO, el objetivo del controlador DMPC es encontrar los coeficientes Laguerre $\eta$ que minimicen la función costo, (39)

$$
\begin{aligned}
& J=\eta^{T} \Omega \eta+2 \eta^{T} \Psi x\left(k_{i}\right) \\
& \text { donde, } \\
& \qquad=\left(\sum_{i=0}^{N_{p-1}} A^{N_{p-i-1}} B L(i)^{T}\right) \\
& \Omega=\left(\sum_{m=1}^{N_{p}} \emptyset(m) Q \emptyset(m)^{T}+R_{L}\right) \\
& \quad \Psi=\left(\sum_{m=1}^{N_{p}} \emptyset(m) Q A^{m}\right)
\end{aligned}
$$

$Q=C^{T} C$ y $R_{L}$ es un escalar que penaliza la acción de control. Cuando el controlador no tiene restricciones y asumiendo que $\Omega^{-1}$ existe cuando $d j / d \eta=0$, la solución óptima para $\eta$, se obtiene analíticamente como (43)

$$
\eta=-\Omega^{-1} \Psi x\left(k_{i}\right)
$$

En la práctica todos los procesos están sujetos a restricciones, por ejemplo, los actuadores tienen un campo limitado de acción, así como una determinada velocidad de cambio (slewrate), además el proceso presenta un rango de operación para cada una de las variables que intervienen, esto hace necesario la introducción de las restricciones en la función a minimizar.

\subsection{Diseño del controlador DMPC}

La implementación del controlador DMPC se divide en dos etapas: la fase de inicialización y la fase de ejecución o proceso iterativo. En la fase de inicialización se obtiene el modelo en espacio de estados en tiempo discreto de la planta y se calculan las matrices constantes $A, B, C, L(0)^{T}, \emptyset, \Omega$, y $\Psi$. Estas matrices se evalúan una sola vez, es decir, antes de entrar al lazo de control. Para determinar los coeficientes de Laguerre se debe establecer previamente el número de términos $(N)$, el factor de escalamiento $(\alpha)$ y el horizonte de predicción $\left(N_{P}\right)$, para lo cual se evalúa la respuesta a una entrada escalón al modificar cada variable. En la Fig. 6 se observa la respuesta transitoria de la velocidad del generador, dado un setpoint de $1000 \mathrm{rpm}$, para dos valores diferentes de $N$, fijando $\alpha=0.8$ y $N_{p}=20$. En el caso de $N=1$, la variable presenta un retardo de 120 segundos y para $N=2$ es de 40 segundos. Para $N$ mayor a tres el sistema no se estabiliza.

Fig. 6. RESPUESTA TRANSITORIA PARA DOS VALORES DIFERENTES DEL NÚMERO DE FUNCIONES DE LAGUERRE

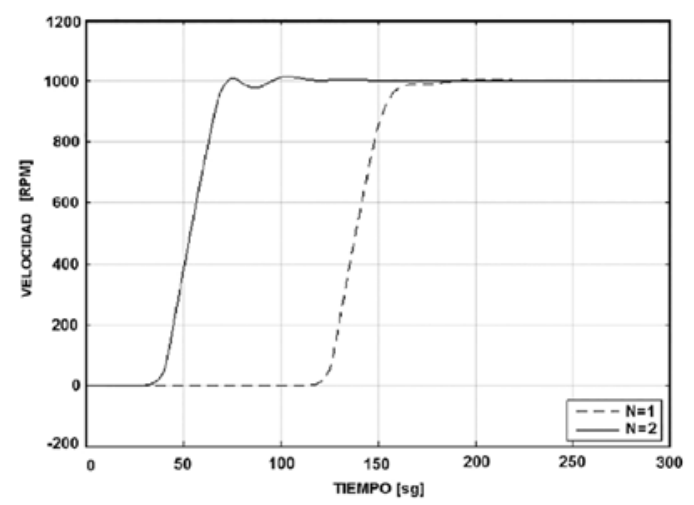

Fuente: Los autores.

Definido el número de funciones de Laguerre ( $N=2$ ) y fijando $N_{p}=20$, se modifica el factor de escalamiento $\alpha$. Al disminuir este paráme- 
tro el tiempo de establecimiento aumenta, Fig. 7, presentando una buena respuesta con $\alpha=0.9$. Al modificar el horizonte de predicción, se observa que al aumentar $N_{p}$ el tiempo de establecimiento aumenta Fig. 8.

Fig. 7. RESPUESTA TRANSITORIA PARA TRES VALORES DIFERENTES DEL FACTOR DE ESCALAMIENTO

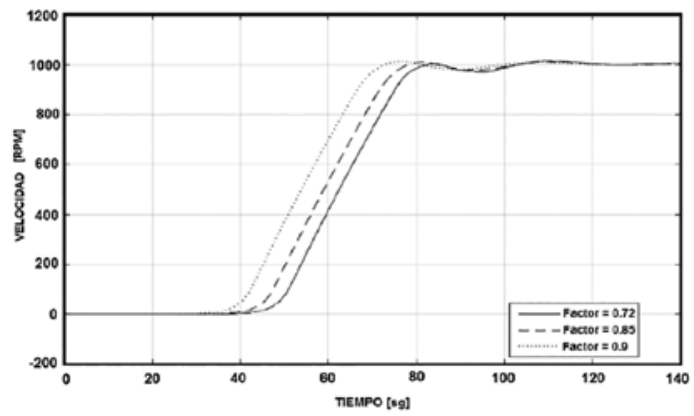

Fuente: Los autores.

Fig. 8. RESPUESTA TRANSITORIA PARA TRES VALORES DIFERENTES DEL HORIZONTE DE CONTROL

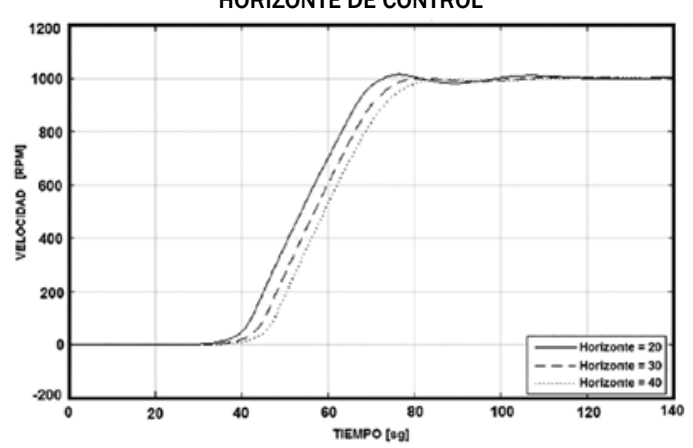

Fuente: Los autores.

Se establecen como parámetros de diseño $N=2, \alpha=0.9, N_{p}=20$ y se calcula la matriz $L(0)^{T}=[0.43588,-0.3923]$. A partir del modelo aumentado y fijando $R_{L}=0.001$ se determina las matrices $\emptyset, \Omega$ y $\Psi$ (44), (45) y (46). Para implementar la estrategia de control predictivo es necesario disponer de la medición de todo el vector de estados, por lo tanto, se emplea el estimador de Kalman, el cual presenta una buena velocidad de convergencia.

$$
\begin{gathered}
\emptyset=\left[\begin{array}{cc}
0.000006 & -0.000002 \\
-0.002317 & -0.000010 \\
0.071815 & 0.164472 \\
0.735866 & 0.174524 \\
7.373417 & -2.764404
\end{array}\right] \\
\Omega=\left[\begin{array}{cc}
172.8821 & -85.3635 \\
-85.3635 & 47.2629
\end{array}\right]
\end{gathered}
$$

$$
\begin{gathered}
\Psi\left[\begin{array}{ccc}
27.3263 & 1058.4748 & 617.24201 \\
-14.8431 & -574.9489 & -339.48399 \\
877.2666 & 29.56302 \\
-517.51101 & -19.2915
\end{array}\right] \quad(46)
\end{gathered}
$$

Para la fase de ejecución el algoritmo predictivo interactúa con la planta y calcula analíticamente la solución óptima para $\eta$. El algoritmo contempla las siguientes restricciones: límites de la variable por controlar $y(k)$ y delta de la señal de control $\Delta u(k)$. En la muestra $k_{i}$, la predicción del estado $x\left(k_{i}+1 \mid k_{i}\right)$ es descrito por la relación (47)

$$
x\left(k_{i}+1 \mid k_{i}\right)=A x\left(k_{i}\right)+B L(0)^{T} \eta
$$

La salida queda definida de la forma (48)

$y\left(k_{i}+1 \mid k_{i}\right)=C A x\left(k_{i}\right)+C B L(0)^{T} \eta$

Si la variable de salida esta limitada $y^{\min } \leq y(k) \leq y^{\max }$, existen tres posibles soluciones para este caso:

- Si $y^{\min }<C A x\left(k_{i}\right)+C B L(0)^{T} \eta<y^{\max }$, entonces $\eta=-\Omega^{-1} \Psi x\left(k_{i}\right)$ es la solución óptima.

- Si $C A x\left(k_{i}\right)+C B L(0)^{T} \eta \leq y^{\min }$, se debe determinar una nueva expresión para $\eta$. Definiendo la variable $M_{a c t}=-C B L(0)^{T}$, la solución óptima del parámetro $\eta$ esta definido por (49)

$$
\eta=-\Omega^{-1}\left(\Psi_{x}\left(k_{i}\right)+M_{\text {act }}^{T} \lambda_{a c t}\right)
$$

donde el multiplicador de Lagrange, $\lambda_{\text {act }}$, es igual a (50):

$$
\begin{aligned}
& \lambda_{a c t}=-\left(M_{a c t} \Omega^{-1} M_{a c t}^{T}\right)^{-1} \\
& \left(-y^{\min }+C A x\left(k_{i}\right)+M_{a c t} \Omega^{-1} \Psi x\left(k_{i}\right)\right)
\end{aligned}
$$

Del mismo modo, si se infringe la restricción del límite superior, entonces la solución analítica para el multiplicador de Lagrange se expresa como (51)

$$
\begin{aligned}
& \lambda_{a c t}=-\left(M_{a c t} \Omega^{-1} M_{a c t}^{T}\right)^{-1} \\
& \left(y^{\max }-C A x\left(k_{i}\right)+M_{a c t} \Omega^{-1} \Psi x\left(k_{i}\right)\right)
\end{aligned}
$$

donde $M_{a c t}=C B L(0)^{T}$. La solución óptima de $\eta$ está dada por la ecuación (49). 
Si el delta de la acción de control se encuentra limitado, $\Delta u^{\min } \leq \Delta u(k) \leq \Delta u^{\max }$, se presenta tres casos:

- Si $\Delta u^{\min } \leq L(0)^{T} \eta \leq \Delta u^{\max }$, entonces la solución óptima, $\eta=-\Omega^{-1} \Psi x\left(k_{i}\right)$, es la solución que minimiza la función de costo $J$.

- Si $L(0)^{T} \eta \leq \Delta u^{\min }$, entonces la solución óptima es la que conduce a $\Delta u\left(k_{i}\right)=\Delta u^{\min }$.

- Si $L(0)^{T} \eta \leq \Delta u^{\max }$, entonces la solución óptima es la que conduce a $\Delta u\left(k_{i}\right)=\Delta u^{\max }$..

Una vez evaluado $\Delta u\left(k_{i}\right)$, la acción de control es $u\left(k_{i}\right)=u\left(k_{i}-1\right)+\Delta u\left(k_{i}\right)$.

\section{RESULTADOS}

Un sistema de control distribuido o DCS es un sistema de control que tiene elementos inteligentes, que se pueden ubicar en distintos niveles de jerarquía, y que no solo reciben acciones o envían variables de proceso, sino que también ejecutan mandos de operación. Los DCS se encargan de descentralizar las labores que tendría un controlador principal. Emerson Delta $V$ es un sistema DCS que ofrece un software y hardware de control avanzado para plantas industriales. El sistema Delta $V$ se puede implementar tanto en plantas discretas como analógicas, trabajando con diferentes protocolos de comunicación. Los dispositivos de control se interconectan en forma de una red conectada a un sistema remoto de adquisición de datos (SCADA) que se encarga de la supervisión. Además, el usuario puede ingresar los valores deseados de las variables y realizar el monitoreo del proceso por medio de una HMI (Human Machine Interface). Para las etapas de programación, configuración de la instrumentación, sintonización de lazos y administración del sistema, Delta $V$ cuenta con una diversidad de software enlazados entre sí, que permiten el cumplimiento de estas funciones. Entre las herramientas de Delta V, se encuentra Control Studio, la cual permite crear estrategias de control como pequeños componentes modulares independientes que se ejecutan simultáneamente. Por medio de esta herramienta se programan las estrategias de control LQG y DMPC, ejecutando cada módulo con un período de muestreo de un segundo. Para analizar la respuesta transitoria de cada controlador se modifica la señal de referencia en un rango de cero a 1500 RPM (Fig. 9 y Fig. 10). En general, para las dos estrategias de control, se observa que la señal de velocidad de la turbina oscila alrededor de la señal de referencia, esto se debe a que la presión del vapor en la tubería no es constante debido al control on-off que tiene la caldera para regular esta variable.

Fig. 9. RESPUESTA TRANSITORIA DEL CONTROLADOR LQG. A) VELOCIDAD DEL GENERADOR B) PORCENTAJE DE APERTURA DE LA VÁLVULA
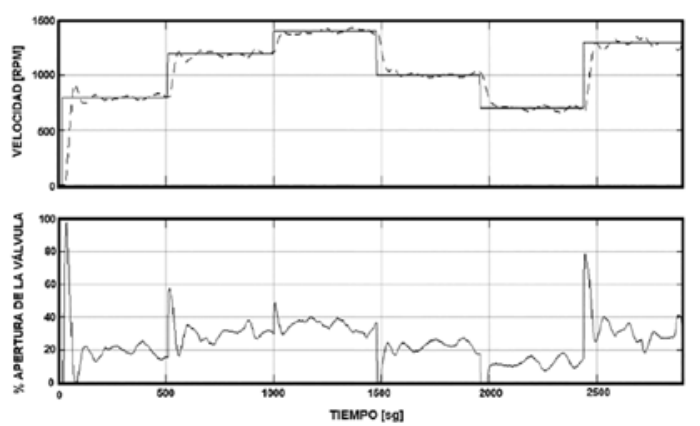

Fuente: Los autores.

Fig. 10. RESPUESTA TRANSITORIA DEL CONTROLADOR DMPC. A) VELOCIDAD DEL GENERADOR B) PORCENTAJE DE APERTURA DE LA VÁLVULA

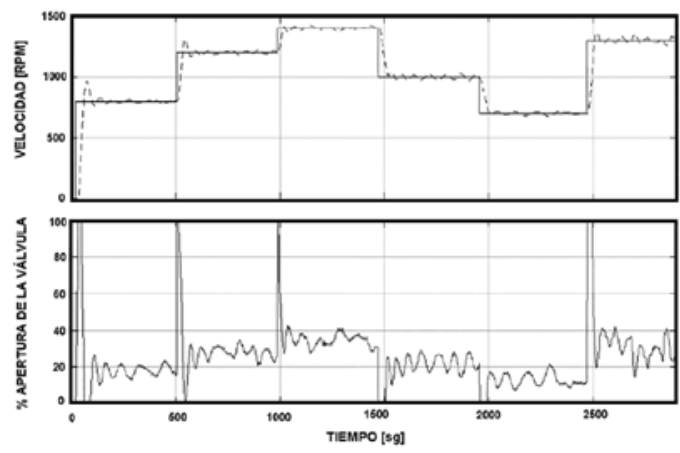

Fuente: Los autores.

La Fig. 11 muestra el comportamiento transitorio de la señal de presión a la entrada de la turbina, cuando el controlador modifica el porcentaje de apertura de la válvula, la presión sigue estos cambios y oscila alrededor de su punto de equilibrio. El control DMPC disminuye este efecto y presenta un menor tiempo de establecimiento comparado con el controlador LQG, esto se debe a que la acción de control del regulador DMPC presenta una tasa de cambio mayor comparada con el controlador LQG. El control DMPC presenta un error de \pm 30 RPM y el control LQG un error de \pm 22 RPM, pero el sobrepaso es mayor en la primera estrategia. Los tiempos de establecimiento 
son cercanos a los valores establecidos en la etapa de diseño.

Fig. 11. PRESIÓN DEL VAPOR A LA ENTRADA DE LA TURBINA

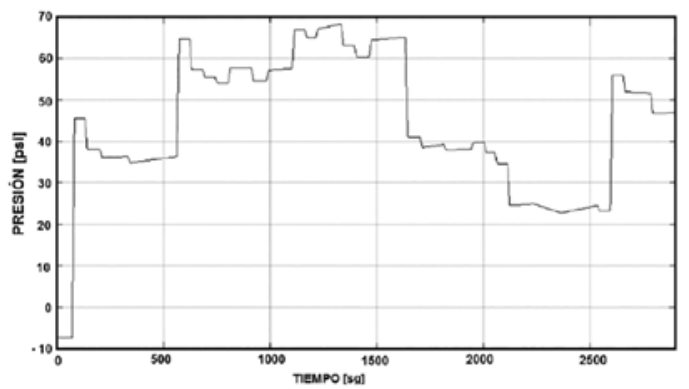

Fuente: Los autores.

Se analizó la robustez de los dos controladores ante cambios de la resistencia del bobinado de campo del generador DC. Al disminuir la resistencia de campo la velocidad del generador debe aumentar, por lo tanto, el controlador debe disminuir el porcentaje de apertura de válvula con el objetivo de mantener la velocidad cercana al nivel de referencia. Se establece una referencia de 1000 RPM y se modifica la resistencia de campo en $t=420$ segundos, $t=950$ segundos y $t=1400$ segundos. En el primer cambio, la resistencia se reduce a un $60 \%$ de su valor nominal, luego se fija a un $20 \%$ del valor nominal $y$, posteriormente, retorna la resistencia al valor nominal. En las figuras 12 y 13 se observa la respuesta de cada controlador. Nuevamente el controlador DMPC presenta un comportamiento más agresivo en la acción de control, lo cual se refleja en una menor desviación que tiene la velocidad del generador con relación al setpoint, especialmente cuando la resistencia cambió del $20 \%$ a su valor nominal.

Fig. 12. RESPUESTA TRANSITORIA DEL CONTROLADOR LQG ANTE CAMBIOS EN LA RESISTENCIA DE CAMPO DEL GENERADOR
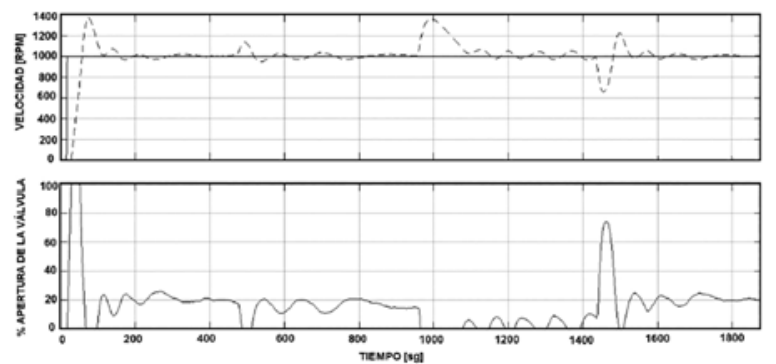

Fuente: Los autores.
Fig. 13. RESPUESTA TRANSITORIA DEL CONTROLADOR DMPC ANTE CAMBIOS EN LA RESISTENCIA DE CAMPO DEL GENERADOR

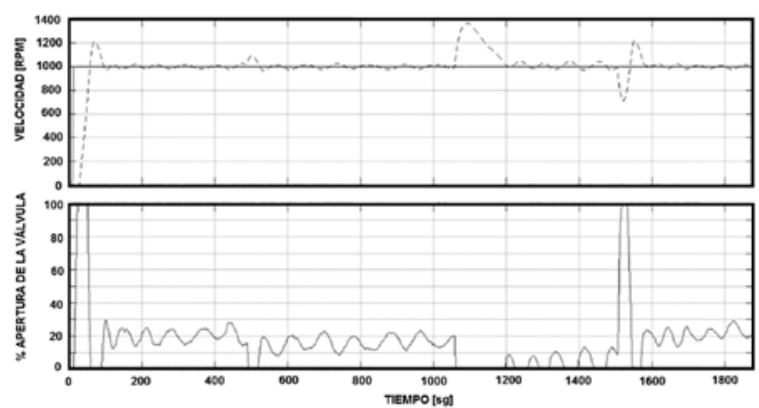

Fuente: Los autores

\section{CONCLUSIONES}

La determinación del modelo dinámico de un sistema es importante a la hora de diseñar el controlador, esto garantiza estabilidad y robustez ante señales de perturbación. Los dos controladores presentan una buena respuesta transitoria ante cambios en la señal de referencia, estableciéndose más rápido el controlador DMPC, pero con una acción de control más agresiva. Las variaciones de la presión en la línea de vapor no afectan la estabilidad del sistema, además el controlador compensa los cambios de la resistencia de campo del generador de corriente continua, lo cual demuestra que las dos estrategias son robustas ante la presencia de perturbaciones.

\section{REFERENCIAS}

[1] Ç.L. Hubka y P. Skolnik, "Steam turbine and steam reheating simulation model". International Conference on Process Control, Junio, 2003. DOI: 10.1109/ PC.2013.6581378.

[2] P. Jiang, L. Gao y Y. Dai, "A new non-linear model of steam turbine unit for dynamic analysis of power system". International Conference on Power System Technology, Octubre, 2010. DOI: 10.1109/POWERCON.2010.5666735.

[3] G. Han, L. Chen, J. Shao y Z. Sum, "Study of fuzzy PID controller for industrial steam turbine governing system". International Symposium on Communications and Information Technology, Octubre, 2005. DOI: 10.1109/ISCIT.2005.1567100.

[4] I. Mohamed, "Adaptation of PID controller using Al technique for speed control of isolated steam turbine". Conference on Electronics, Communications and Computers, Marzo, 2012. DOI: 10.1109/JECECC.2012.6186962.

[5] H. Reza y R. Mohseni, "Generalized Model Predictive Control for a Multivariable Boiler- Turbine Unit". 11th International Conference on Control, Automation and Systems, Octubre, 2011. ISSN: 2093-7121. 
[6] M. Kordestani, M. Soleimani y A. Mirzaee, "Predictive control of large steam turbines". 9th Asian Control Conference, Junio, 2013. DOI: 10.1109/ ASCC.2013.6606366.

[7] B. Codrons, "LQG control of steam temperature in power plants". European Control Conference, Septiembre, 2003. Print ISBN: 978-3-9524173-7-9.

[8] M. Dulau y D. Bica, "Mathematical modelling and simulation of the behavior of the steam turbine". 7th International Conference Interdisciplinarity in Engineering, Octubre, 2013. DOI: 10.1016/j.protcy.2013.12.555.

[9] S. Chapman, Máquinas Eléctricas. McGraw Hill. ISBN 958-600-125-3, Segunda edición, pp. 283-290, 1993.
[10] K. Ogata, Sistemas de control en tiempo discreto. Ed. Pearson Educacion. ISBN 0-13-034281-5, pp. 596609, 1995.

[11] R. Burns, Advanced Control Engineering. Ed. Butterworth Heinemann. ISBN 0750651008, pp. 288299, 2001.

[12] X. Lan Li, J. Gyu y H. Shin, "Comparison and evaluation of anti-windup PI Controllers". Journal of Power Electronics, vol. 11 no. 1, Enero, 2011.

[13] L. Wang, Model Predictive Control System Design and Implementation Using MATLAB. ISBN 978-84882330-3, pp. 85-142, 2009. 